\title{
Lemongrass and Ginger Potency for Blood Glucose Control
}

\author{
Claudia Christy $^{1 *}$, Maria D.P.T. Gunawan Puteri ${ }^{1}$, Abdullah Muzi Marpaung ${ }^{1}$ \\ ${ }^{I}$ Department of Food Technology, Faculty of Life Sciences and Technology, Swiss German University, \\ Tangerang 15143, Indonesia \\ *Corresponding author: claudia.christy@student.sgu.ac.id
}

\begin{abstract}
Lemongrass (Cymbopogon citratus) and ginger (Zingiber officinale) are herbs that have been used to flavour food and beverages, in addition, they are also believed to possess health benefits. One of them is their ability to control blood glucose levels. Blood glucose control not only is beneficial for those who already have blood glucose regulating problems, but it may also be beneficial for the prevention of blood glucose-related diseases such as type 2 diabetes. The purpose of this research is to determine lemongrass and ginger potency for blood glucose control as well as the various populations that will benefit from blood glucose control. This research was conducted using literature reviews of various journals. From this study, it was found that lemongrass and ginger have a good potency in blood glucose control and are able to benefit people who are interested in diabetes prevention, those who are at high risk to developing diabetes, PCOS patients, people with skin concerns, and people with poor mood and/or energy levels.
\end{abstract}

Keywords: Lemongrass, Ginger, Blood glucose control, Diabetes prevention, Cymbopogon citratus, Zingiber officinale.

\section{INTRODUCTION}

In order for the body to maintain its normal bodily functions, the body functions on negative feedback in order to keep blood sugar levels at a normal level [1]. Blood sugar levels are considered normal when it is below $100 \mathrm{mg} / \mathrm{dL}$ when fasting and is less than 140 $\mathrm{mg} / \mathrm{dL}$ two hours after eating [2]. After eating, glucose levels in the blood rise triggering the release of the hormone called insulin produced by the pancreas. Insulin is responsible for facilitating glucose uptake to cells and when in excess, this hormone signals glycogen synthesis as a form of storage for glucose. When blood glucose levels are low, the pancreas releases a hormone called glucagon which releases glucose to the bloodstream [1].

However, an unhealthy lifestyle may cause this function to be defected [3]. For example, when blood glucose levels are consistently high due to things such as the frequent consumption of food high in refined carbohydrates, it causes the constant production of insulin to the bloodstream. When this occurs frequently, the body may develop insulin resistance [3], where the cells are unable to respond to insulin, causing the inability of glucose to enter the bloodstream which leads to blood sugar levels to be above normal [4]. This condition is called hyperglycaemia, and the inability to respond normally to insulin is called type 2 diabetes [5].

Anyone could be at risk of developing this condition, hence why it is important to prevent blood sugar levels from having spikes or is often at a higher range to reduce the risk of developing type 2 diabetes [6]. In addition, good blood glucose control has been shown to improve certain health conditions such as PCOS, acne, as well as improvement of mood and energy.

There are various ways in order to maintain a healthy blood sugar level, including adapting a healthy diet, regular exercise, and being a healthy weight [7]. Medications are also prescribed in order to keep blood sugar in check for people with diabetes as well as in prevention [8], [9]. In Indonesia, people often utilize herbal remedies for its various health benefits which includes lowering high blood sugar. Various herbal plants have been believed to have benefits in controlling high blood glucose levels based on 
anecdotal evidence [10]. In the modern age, more herbals are being analysed scientifically to determine whether herbal plants are indeed able to manage blood sugar levels [11].

Cymbopogon citratus and Zingiber officinale, or more commonly known as lemongrass and ginger respectively are examples of popular plants that have been utilized in Indonesia to flavour food as well as ingredients used in beverages, they are often used together due to their complementary flavour profile. They are also believed to have health benefits and one of them is in lowering high blood sugar levels. The long history of usage for both species as food and medicinal treatment have also shown its potency in terms of safety. However, in order to commercialize a product with claims of controlling high blood sugar legally, there has to be scientific evidence to back up those claims [12]. Hence why it is important to provide research that supports its efficacy and safety.

Safety and efficacy are often a point of consideration in the commercialization of synthetic drugs. In the utilisation of lemongrass and gingerbased products with health claims, its safeness as well as its efficacy should also be evaluated.

\section{METHOD}

Papers included were searched using Google Scholar. The literature review includes how blood sugar control can benefit non-diabetic individuals, effect of glycemic levels in increasing or decreasing risk of diabetes, effect of glycemic levels in increasing or decreasing complications in PCOS patients, and types of population that are at a higher risk of developing diabetes.

For lemongrass and ginger safety and efficacy analysis, papers used were both in-vitro and in-vivo studies. In-vitro studies included the <-amylase inhibitory and/or <-glucosidase activity of Zingiber officinale and Cymbopogon citratus or its bioactive components in order to display its blood glucose lowering capability. For in-vivo studies, papers that were included showed that Cymbopogon citratus and Zingiber officinale or its bioactive compounds are able to significantly lower blood glucose levels or improve sensitivity.

If the level of reduction in blood glucose levels was not included in the study, it was determined by using the formula:

$\%$ blood glucose reduction $=\frac{(\text { Final }- \text { initial })}{\text { Final } x 100 \%}$
Notes:

Final : final blood glucose level of treated subject

Initial : blood glucose level before treatment or of untreated subject (depending on the study)

Insulin levels was also calculated the same way where:

Final : final insulin level of treated subject

Initial : insulin level before treatment or of untreated subject (depending on the study)

Calculation of HOMA-IR to display improvement in insulin sensitivity is also using the same formula:

Final : final HOMA-IR value of treated subject

Initial : HOMA-IR value before treatment or of untreated subject (depending on the study)

\section{RESULTS AND DISCUSSION}

\subsection{Benefits of blood glucose control in healthy individuals}

Blood sugar control is crucial in the prevention of type 2 diabetes [6], not only that, it has other health benefits as well. Blood sugar control can have an impact on mood and energy levels. This is shown in a study where participants that were put on low glycemic diets had lower fatigue, total mood disturbance, and depression symptoms compared to those put on a high glycemic diet [13].

Furthermore, in a study done by Levitan et al., in 2004 , it is found that individuals with higher post challenge glucose levels is at higher risk of developing cardiovascular heart disease (CVD) compared to the group that had the lowest post challenge glucose levels, indicating non-diabetic hyperglycaemia may be a risk factor of CVD [14].

In relation to skin health, one of the possible side effects of insulin resistance and hyperinsulinemia is Acanthosis nigricans. Although often observed in people with diabetes, this condition may also affect relatively healthy people and may appear in kids, but it is more prevalent in adults [15]. A clinical trial done on a 27 years old male with obesity showed that improvement in insulin resistance through diet control helps with in treating AN [16]. In addition, in a review that looks at case reports and clinical trials, it was shown that the administration of metformin drugs is effective in the treatment of AN [17]. 
High glycaemic diets that lead to the large productions of insulin and insulin growth factors (IGF) may also exacerbate skin problems such as excess sebum production and acne [18]. Few studies have shown that a high glycemic diet that causes blood sugar spikes resulted in the increased production of acne and sebum production [19], [20]. The high levels of IGF are able to bind to the receptors in the pores enlarging the oil gland which elevates oil production and inflammatory mediators [19]. Moreover, this IGF also elevates the androgen hormones that also triggers the receptors in the pores [21]. Furthermore, some studies have shown that taking diabetic drugs, namely metformin, is able to lower blood glucose levels showed an improvement of acne in acne patients [22]. This indicates that people with acne or excessive oil production may benefit from controlling high blood sugar levels.

\subsection{Benefits of blood glucose control in PCOS patients}

Elevated levels of inflammation due to oxidative stress in the body directly causes the ovary to produce excess androgen [21]. Inflammation in the body may be triggered from diet such as glucose or increased adipose deposits in the abdominal region. Moreover, inflammation triggered by the ingestion of glucose is linked to insulin resistance [21]. It is important for PCOS patients to regulate blood sugar levels in order to prevent hyperglycemia.

To emphasize the importance of glycemic control, a 2015 research showed that hyperglycemia effects glutathione peroxidase activity, which indicates the increase susceptibility to oxidative stress in non-obese women with PCOS [23]. Moreover, oxidative stress is the marker for insulin resistance and testosterone levels [23].

In a 2019 study, the result showed that obese women put on glycemic control through a low glycemic diet exhibited an improvement in insulin sensitivity, hyperandrogenism, hirsutism, acne, and menstrual irregularities [24]. In a double-blind, placebo-controlled study of women with PCOS, the administration of metformin improved pregnancy rates and live-birth rates, it is also found that it showed an even more significant improvement in obese PCOS patients [25]. Moreover, acarbose, an alpha glucosidase inhibitor, is able to reduce testosterone, TG, and VLDL, and increase HDL in the treatment of PCOS as shown from a meta-analysis data [26].
Since insulin resistance is mainly caused due to poor blood sugar levels control, taking care of it may reduce the risk of PCOS in women [27]. Other than that, since up to $70 \%$ of PCOS patients have insulin resistance [27], it is also important for them to control their blood sugar levels in order to prevent serious complications such as the development of type 2 diabetes (Diamanti-Kandarakis \& Dunaif, 2012).

\subsection{Benefits of blood glucose control in people with higher risk of developing type 2 diabetes}

Though everyone can benefit from controlling blood glucose levels to prevent type 2 diabetes, people who are obese, elders, or have a family history of diabetes are at higher risk to developing this disease hence they may obtain experience the benefit more significantly than those who doesn't have these conditions.

Several studies have shown obese or overweight people are at higher risk of developing insulin resistance making it difficult for them to control blood sugar levels [29]-[31]. According to a research, this is because in people who are overweight or obese, there is an excess in visceral fat and increased inflammation which are linked to insulin resistance [30]. This is further supported by another study that observed that there is correlation between weight gain in adults and insulin resistance mediated by visceral and liver fat [29].

Elders are at higher risk of blood sugar related diseases as with age, some people's ability to regulate blood sugar levels decline [32]. This is further supported by another study that looks at carbohydrate metabolism in elderlies, it was found that aging increases glucose intolerance [33]. Hence why it is important for elders to monitor their blood sugar levels, however, studies show elderlies may find it difficult to stay physically active [34]. This is where they may benefit from consuming herbal products to help lower high blood sugar levels.

It was also found in a study, that people who have a family history of diabetes also are at higher risk of developing said diseases, though the specific reason is not completely understood, it is mainly due to genetics [35]. Those who have family with diabetes should take measures necessary in order to control blood sugar levels [5]

\subsection{Lemongrass in blood glucose control}

Lemongrass also has shown promising AGI activity [36] and blood glucose lowering capabilities 
[37]. Table 1 displays various studies done that analyses the effectiveness, mechanism, and also the method of the research of various parts of lemongrass in glycemic control.

Various lemongrass extracts and its bioactive components have shown their ability in lowering blood glucose levels significantly in studies done on both diabetic [38] and healthy animals [39] even though the results were often not significant. There are also several in-vitro studies displaying its AGI and AAI activities. In all relevant studies, the administration of lemongrass increased insulin sensitivity [37], [40]. In some studies, however, some found that the administration of lemongrass and its bioactive effects had an impact on either increasing [41] or decreasing insulin levels [40].

Upon a closer look at the research, it can be seen that in studies that reported a rise of insulin levels, its untreated diabetic control had insulin levels lower than non-diabetic control. The treatment only raised insulin levels closer to those of the control animals [37], [41]. Moreover, in the study where the healthy mice were given lemongrass, its insulin did not change significantly [37].

On the other hand, where the diabetic untreated animals had higher insulin levels above its healthy control counterpart, the administration of lemongrass decreased insulin levels that are high to begin with, bringing the value closer to control [40]. This may occur due to the effect of lemongrass in increasing insulin sensitivity, hence making the body require less insulin in signalling the cells [40]. These findings suggest that, depending on the type of diabetes, the body is able to respond accordingly to the effects of Cymbopogon citratus.

In the studies, there were no reported adverse effects observed towards the test subjects. Furthermore, the specific safety of Cymbopogon citratus can be seen on table 2. Based on toxicology reports, it can be concluded that lemongrass is indeed safe and has no adverse effects in long term use. Based on toxic levels of substances, Cymbopogon citratus is not near what is considered harmful. The amount of lemongrass consumed to have negative effects only happens at very high concentrations of it. Moreover, lemongrass has been used in various food and beverage products for a long time, and the National Agency of Drug and Food Control also considered it to be safe and allowed as ingredients in products [42]. So, it can be said that lemongrass is safe to be consumed over long periods

Table 1 Lemongrass blood glucose controlling capabilities

\begin{tabular}{|c|c|c|c|c|c|}
\hline Preparation & Model & Mechanism & Efficacy & Dose & References \\
\hline $\begin{array}{l}\text { Aqueous extraction } \\
\text { with heat }\end{array}$ & Healthy Mice & Hypoglycemic effect & $\begin{array}{c}\text { Fasting blood glucose } \\
26.1 \% \text { lower than } \\
\text { control }\end{array}$ & $\begin{array}{l}500 \mathrm{mg} / \mathrm{kg} \\
\text { day }\end{array}$ & [43] \\
\hline Essential oil & In Vitro & $\langle$-amylase inhibition & $\begin{array}{l}\text { IC50(maltose): } 6.97 \\
(\mathrm{~L} / \mathrm{mL}\end{array}$ & - & [44] \\
\hline Aqueous maceration & In Vitro & $\langle$-glucosidase inhibition & $\begin{array}{c}\text { IC50 (sucrase): } 132.89 \\
\mathrm{mg} / \mathrm{mL}\end{array}$ & - & \multirow[t]{5}{*}{ [45] } \\
\hline $\begin{array}{c}\text { Aqueous extraction } \\
\text { with heat }\end{array}$ & In Vitro & $\langle$-glucosidase inhibition & $\begin{array}{l}\text { IC50(sucrase): } 14.46 \\
\mathrm{mg} / \mathrm{mL}\end{array}$ & - & \\
\hline $\begin{array}{c}\text { Aqueous extraction } \\
\text { with heat, spray dried }\end{array}$ & In Vitro & $\langle$-glucosidase inhibition & $\begin{array}{l}\text { IC50(sucrase): } 18.22 \\
\mathrm{mg} / \mathrm{mL}\end{array}$ & - & \\
\hline \multirow[t]{2}{*}{ Spray dried } & Diabetic Mice & Improve maltose tolerance & $\begin{array}{c}\text { LG AUC: } 14472 \pm \\
3205 \mathrm{mg} \mathrm{min} / \mathrm{dl} \\
\text { Control AUC: } 24349 \pm \\
2878 \mathrm{mg} \mathrm{min} / \mathrm{dl}\end{array}$ & $\begin{array}{c}4.33 \mathrm{~g} / \mathrm{kg} \\
\mathrm{BW}\end{array}$ & \\
\hline & & $\begin{array}{l}\text { Improve sucrose and } \\
\text { glucose tolerance }\end{array}$ & $\begin{array}{c}\text { Sucrose } \\
\text { LG AUC: } 17037 \pm \\
1875 \mathrm{mg} \text { min/dl } \\
\text { Control AUC: } 22739 \pm \\
2343 \mathrm{mg} \mathrm{min} / \mathrm{dl} \\
\text { Glucose } \\
\text { LG AUC: } 16034 \pm \\
2045 \mathrm{mg} \mathrm{min} / \mathrm{dl} \\
\text { Control AUC: } 25530 \pm \\
1924 \mathrm{mg} \mathrm{min} / \mathrm{dl}\end{array}$ & $\begin{array}{l}6.67 \mathrm{~g} / \mathrm{kg} \\
\mathrm{BW}\end{array}$ & \\
\hline $\begin{array}{l}90 \% \text { ethanolic } \\
\text { maceration }\end{array}$ & Healthy Mice & Hypoglycemic & $\begin{array}{l}\text { Blood glucose } 23.58 \% \\
\text { reduced by day } 30\end{array}$ & $\begin{array}{c}200 \mathrm{mg} / \mathrm{kg} \\
\mathrm{BW}\end{array}$ & \multirow[t]{2}{*}{ [39] } \\
\hline Aqueous maceration & Healthy Mice & Hypoglycemic & $\begin{array}{l}\text { Blood glucose } 21.59 \% \\
\text { reduced by day } 30\end{array}$ & $\begin{array}{c}200 \mathrm{mg} / \mathrm{kg} \\
\mathrm{BW}\end{array}$ & \\
\hline
\end{tabular}




\begin{tabular}{|c|c|c|c|c|c|}
\hline $\begin{array}{l}50 \% \text { methanolic } \\
\text { maceration }\end{array}$ & In Vitro & <-glucosidase inhibition & $\begin{array}{l}73 \% \text { sucrase inhibition } \\
\text { at } 0.02 \mathrm{mg} / \mathrm{mL}\end{array}$ & - & {$[46]$} \\
\hline $\begin{array}{l}\text { Aqueous extraction } \\
\text { with heat, pre- } \\
\text { evaporation }\end{array}$ & In Vitro & $\begin{array}{l}\text { <-glucosidase inhibition \& } \\
\text { <-amylase inhibition }\end{array}$ & $\begin{array}{l}\text { AGI: } 100 \% \text { sucrase } \\
\text { inhibition at } 0.3 \mathrm{~g} / \mathrm{mL} \\
\text { AAI: } 81.77 \% \text { inhibition } \\
\text { at } 0.3 \mathrm{~g} / \mathrm{mL}\end{array}$ & - & \multirow[t]{2}{*}{ [47] } \\
\hline $\begin{array}{l}\text { Spray dried aqueous } \\
\text { extract }+ \text { maltodextrin } \\
+ \text { Arabic gum }\end{array}$ & In Vitro & $\begin{array}{c}\langle\text {-glucosidase inhibition \& } \\
\text { <-amylase inhibition }\end{array}$ & $\begin{array}{c}\text { AGI: } 61.69 \% \text { sucrase } \\
\text { inhibition at } 0.3 \mathrm{~g} / \mathrm{mL} \\
\text { AAI: } 49.49 \% \text { inhibition } \\
\text { at } 0.3 \mathrm{~g} / \mathrm{mL}\end{array}$ & - & \\
\hline Aqueous maceration & In Vitro & <-glucosidase inhibition & $\begin{array}{c}\text { IC50 (maltase): } 302.27 \\
\text { mg/mL }\end{array}$ & - & \multirow[t]{3}{*}[48]{} \\
\hline \multirow[t]{2}{*}{$\begin{array}{l}96 \% \text { ethanolic } \\
\text { maceration }\end{array}$} & In Vitro & <-glucosidase inhibition & $\begin{array}{c}\text { IC50 (sucrase): } 8.74 \\
\text { mg/mL }\end{array}$ & - & \\
\hline & & $\langle$-glucosidase inhibition & $\begin{array}{c}\text { IC50 (maltase): } 18.93 \\
\mathrm{mg} / \mathrm{mL}\end{array}$ & - & \\
\hline $\begin{array}{l}\text { Aqueous extraction } \\
\text { with heat of: }\end{array}$ & & & & & \multirow[t]{5}{*}[49]{} \\
\hline Fresh lemongrass & \multirow[t]{4}{*}{ In Vitro } & \multirow[t]{4}{*}{$\langle$-glucosidase inhibition } & $\begin{array}{c}\text { IC50 (sucrase): } 17.93 \\
\mathrm{mg} / \mathrm{mL}\end{array}$ & - & \\
\hline Dried lemongrass & & & $\begin{array}{c}\text { IC50 (sucrase): } 24.5 \\
\mathrm{mg} / \mathrm{mL}\end{array}$ & - & \\
\hline $\begin{array}{c}\text { Combined } \\
\text { lemongrass and } \\
\text { ginger extract }\end{array}$ & & & $\begin{array}{l}61.74 \% \text { sucrase } \\
\text { inhibition }\end{array}$ & - & \\
\hline $\begin{array}{c}\text { (Lemongrass } 24.5 \\
\mathrm{mg} / \mathrm{mL} \text { and ginger } \\
19.61 \mathrm{mg} / \mathrm{mL} \text {, ratio } \\
1: 1)\end{array}$ & & & $\begin{array}{l}57.77 \% \text { maltase } \\
\text { inhibition }\end{array}$ & - & \\
\hline $\begin{array}{l}\text { Aqueous extraction } \\
\text { with heat: }\end{array}$ & & & & & \multirow[t]{5}{*}[50]{} \\
\hline Sterilized & \multirow[t]{4}{*}{ In Vitro } & \multirow[t]{4}{*}{ <-glucosidase inhibition } & $\begin{array}{l}33.9 \% \text { sucrase } \\
\text { inhibition }\end{array}$ & - & \\
\hline Pasteurized & & & $\begin{array}{l}24.4 \% \text { sucrase } \\
\text { inhibition }\end{array}$ & - & \\
\hline $\begin{array}{l}\text { Pasteurized and } \\
\text { refrigerated }\end{array}$ & & & $\begin{array}{l}69.5 \% \text { sucrase } \\
\text { inhibition }\end{array}$ & - & \\
\hline Spray dried & & & No sucrase inhibition & - & \\
\hline $\begin{array}{l}\text { Aqueous maceration } \\
\text { of: }\end{array}$ & & & & & \multirow[t]{3}{*}[51]{} \\
\hline Lemongrass roots & \multirow[t]{2}{*}{$\begin{array}{c}\text { Dexamethasone } \\
\text { induced diabetic mice }\end{array}$} & \multirow[t]{2}{*}{$\begin{array}{c}\text { Reduce fasting and } \\
\text { postprandial glucose levels }\end{array}$} & $\begin{array}{c}\text { Blood glucose levels } \\
21.8 \% \text { lower by day } 14\end{array}$ & $\begin{array}{c}100 \mathrm{mg} / \mathrm{kg} \\
\text { day }\end{array}$ & \\
\hline Lemongrass flower & & & $\begin{array}{l}\text { Blood glucose levels } \\
3.6 \% \text { lower by day } 14\end{array}$ & $\begin{array}{c}100 \mathrm{mg} / \mathrm{kg} \\
\text { day }\end{array}$ & \\
\hline \multirow[t]{3}{*}{ Lemongrass tea } & Diabetic Mice & \multirow[t]{2}{*}{$\begin{array}{c}\text { Improve glucose tolerance, } \\
\text { insulin sensitivity, } \beta \text {-cell } \\
\text { functions and dyslipidemia } \\
\text { Raise insulin to normal } \\
\text { levels }\end{array}$} & $\begin{array}{l}\text { Reduce Fasting and } \\
\text { postprandial glucose } \\
\text { levels (until 60.3\%) } \\
\text { Insulin levels } 29.17 \% \\
\text { higher than diabetic } \\
\text { control (DC) }\end{array}$ & $\begin{array}{l}250 \mathrm{mg} / 100 \\
\mathrm{~mL} \text { water }\end{array}$ & \multirow[t]{3}{*}[37]{} \\
\hline & Diabetic Mice & & $\begin{array}{c}\text { Reduce Fasting and } \\
\text { postprandial glucose } \\
\text { levels (until } 60.3 \% \text { ) } \\
\text { Insulin levels } 31.35 \% \\
\text { higher than DC }\end{array}$ & $\begin{array}{l}500 \mathrm{mg} / 100 \\
\mathrm{~mL} \text { water }\end{array}$ & \\
\hline & Healthy Mice & - & No significant effect & $\begin{array}{l}500 \mathrm{mg} / 100 \\
\mathrm{~mL} \text { water }\end{array}$ & \\
\hline $\begin{array}{l}\text { Extraction, dried, } \\
\text { diluted in acetone }\end{array}$ & \multirow[t]{5}{*}{ In Vitro } & \multirow[t]{5}{*}{$\begin{array}{c}\text { <-glucosidase inhibition \& } \\
\text { <-amylase inhibition }\end{array}$} & $\begin{array}{l}\text { AGI is at } 1 \mathrm{mg} / \mathrm{mL} \\
\text { AAI in EC50 }\end{array}$ & - & \multirow[t]{5}{*}[52]{} \\
\hline Acetonic extract & & & $\begin{array}{c}\text { AGI: } 70.55 \% \\
\text { AAI: } 0.65 \mathrm{mg} / \mathrm{mL}\end{array}$ & - & \\
\hline Methanolic extract & & & $\begin{array}{c}\text { AGI: } 45.42 \% \\
\text { AAI: } 0.31 \mathrm{mg} / \mathrm{mL} \\
\end{array}$ & - & \\
\hline Ethyl acetate extract & & & $\begin{array}{c}\text { AGI: } 95.02 \% \\
\text { AAI: } 1.2 \mathrm{mg} / \mathrm{mL}\end{array}$ & - & \\
\hline Hexane extract & & & $\begin{array}{c}\text { AGI: } 100 \% \\
\text { AAI: } 1.3 \mathrm{mg} / \mathrm{mL}\end{array}$ & - & \\
\hline Essential oil & $\begin{array}{l}\text { PX-47 induced } \\
\text { diabetic rats }\end{array}$ & $\begin{array}{c}\text { Improve insulin } \\
\text { sensitivity, reduce insulin } \\
\text { levels (closer to normal } \\
\text { control) }\end{array}$ & $\begin{array}{c}\text { Blood Glucose } 29.65 \% \\
\text { lower than diabetic } \\
\text { control } \\
\text { Insulin levels } 12 \% \\
\text { lower than DC } \\
\text { HOMA-IR 19.75\% } \\
\text { lower than DC }\end{array}$ & $\begin{array}{l}400 \mathrm{mg} / \mathrm{kg} \\
\mathrm{BW}\end{array}$ & [40] \\
\hline
\end{tabular}




\begin{tabular}{|c|c|c|c|c|c|}
\hline & & & $\begin{array}{c}\text { Blood Glucose } 47.31 \% \\
\text { lower than DC } \\
\text { Insulin levels } 14 \% \\
\text { lower than DC } \\
\text { HOMA-IR } 65.84 \% \\
\text { lower than DC }\end{array}$ & $\begin{array}{l}800 \mathrm{mg} / \mathrm{kg} \\
\mathrm{BW}\end{array}$ & \\
\hline $\begin{array}{c}\text { Aqueous maceration } \\
\text { (liquid) }\end{array}$ & $\begin{array}{c}\text { Alloxan induced } \\
\text { diabetic rats }\end{array}$ & Anti-hyperglycemia & $\begin{array}{c}\text { Blood glucose } 26.6 \% \\
\text { reduced }\end{array}$ & $\begin{array}{c}1.5 \mathrm{~mL} / 100 \mathrm{~g} \\
\mathrm{BW}\end{array}$ & [53] \\
\hline d-limonene & $\begin{array}{c}\text { Streptozotocin } \\
\text { induced DM1 rats }\end{array}$ & Raise insulin levels & $\begin{array}{c}\text { Blood glucose } 36.41 \% \\
\text { reduced after } 28 \text { days } \\
\text { Insulin 36.3\% higher } \\
\text { than DC } \\
\end{array}$ & $\begin{array}{c}50 \mathrm{mg} / \mathrm{kg} \\
\mathrm{BW}\end{array}$ & [41] \\
\hline Linalool & \multirow[t]{3}{*}{$\begin{array}{l}\text { Streptozotocin } \\
\text { induced DM rats }\end{array}$} & \multirow[t]{3}{*}{ Anti-hyperglycemia } & $\begin{array}{c}\text { Blood Glucose } 28.31 \% \\
\text { reduced }\end{array}$ & $\begin{array}{c}20 \mathrm{mg} / \mathrm{kg} \\
\mathrm{BW}\end{array}$ & \multirow[t]{3}{*}{ [54] } \\
\hline Limonene & & & $\begin{array}{l}\text { Blood Glucose } 25.65 \% \\
\text { reduced }\end{array}$ & $\begin{array}{c}20 \mathrm{mg} / \mathrm{kg} \\
\mathrm{BW}\end{array}$ & \\
\hline Linalool + Limonene & & & $\begin{array}{l}\text { Blood Glucose } 46.15 \% \\
\text { reduced }\end{array}$ & $\begin{array}{c}10 \mathrm{mg} / \mathrm{kg} \\
\mathrm{BW}\end{array}$ & \\
\hline Citral & $\begin{array}{l}\text { Streptozotocin } \\
\text { induced DM rats }\end{array}$ & $\begin{array}{c}\text { AAI \& Anti- } \\
\text { hyperglycemia }\end{array}$ & $\begin{array}{l}\text { Blood Glucose 26\% } \\
\text { reduced }\end{array}$ & $\begin{array}{c}16 \mathrm{mg} / \mathrm{kg} \\
\mathrm{BW}\end{array}$ & [55] \\
\hline Ethanolic extract & $\begin{array}{c}\text { Streptozotocin } \\
\text { induced DM } \\
\text { hyperlipidaemic rats }\end{array}$ & Anti-hyperglycemia & $\begin{array}{l}\text { Blood glucose } 45.18 \% \\
\text { lower than control }\end{array}$ & $\begin{array}{c}1000 \mathrm{mg} / \mathrm{kg} \\
\mathrm{BW}\end{array}$ & [38] \\
\hline
\end{tabular}

Table 2 Lemongrass toxicology results

\begin{tabular}{|c|c|c|c|}
\hline Toxicology & Toxic Level & Method & Reference \\
\hline Acute oral toxicity: $>5000 \mathrm{mg} / \mathrm{kg} \mathrm{BW}$ & $>50<500 \mathrm{mg} / \mathrm{kg}$ & Healthy mice & {$[43],[56]$} \\
\hline $5 \mathrm{~g} / \mathrm{kg}$ b. w. for the oral administration to rats* & - & Healthy rats & {$[57],[58]$} \\
\hline LD50: $2500.20 \mathrm{mg} / \mathrm{kg}$ & LD50: $50-500 \mathrm{mg} / \mathrm{kg}$ & Streptozotocin induced DM hyperlipidaemic rats & {$[38]$} \\
\hline
\end{tabular}

\subsection{Ginger in blood glucose control}

Ginger, similar to lemongrass, has been shown to have antidiabetic activity through alpha glucosidase inhibition and presence $\beta$-Sesquiphellandrene which increases insulin sensitivity [11], [36]. Table 3 shows literature review of the capability of Zingiber officinale in improving glycemic control.

Ginger and its bioactive components have consistently shown that they are efficacious in lowering blood sugar controls through various mechanisms and have AGI or AAI properties. Additionally, in several studies, it was shown that insulin sensitivity is improved through measurement of HOMA-IR [59]-[61]. The improvement in insulin sensitivity resulted in the lowering of circulating insulin levels as the cells no longer require as much insulin to be signalled [59]-[61].

Interestingly, in some cases, ginger also has an effect on increasing insulin secretion where the diabetic control had low insulin levels compared to its non-diabetic control [62]-[64]. The increase of insulin secretion is observed in studies done on diabetic rats or mice. The different result may be caused by the fact that some types of diabetes cause hypoinsulinemia, thus ginger and its bioactive properties were found to help repair this function. In a study by Samad et al., it was found that gingerol, a bioactive substance contained in ginger, improves insulin secretion through activation of GLP-1 and also regulating insulin granule exocytosis [62]. In addition, in the studies by Akhani and Samad et alThe insulin levels measured are postprandial insulin levels instead of fasting, which may be another reason for this contradicting results [62], [64].

Although most significant results are seen in diabetic animals, Zingiber officinale is still able to lower blood sugar levels in healthy [65] and obese [66] test animals, as well as, HF or HFHC diet mice [60], [61]. These findings suggest that ginger is able to benefit people who are healthy, overweight or obese, or have an unhealthy diet.

From the research, there were no reported negative effects of gingers and the toxicology results can be seen on table 4 . The toxicology reports concluded that ginger is generally safe and only unsafe in very high amounts. Even though gingerol seems to be in the harmful range, however the amount of gingerol present in ginger is very little with $75.25 \mathrm{mg} / 100 \mathrm{~g}$ fresh weight [67]. Hence the daily consumption of Zingiber officinale is deemed safe. Moreover, based on the database of the National Agency of Drug and Food Control of Indonesia (BPOM), there are also various products both registered as food and herbal medicine 
containing Zingiber officinale that is consumed on a daily basis [68]. Thus, it can be said that ginger is safe to be used in food and drinks for daily consumption.

Table 3 Ginger blood glucose controlling capabilities

\begin{tabular}{|c|c|c|c|c|c|}
\hline Preparation & Model & Mechanism \& Actives & Efficacy & Dose & References \\
\hline $\begin{array}{l}\text { Ethyl Acetate } \\
\text { Extraction }\end{array}$ & In vitro & $\begin{array}{l}\text { Gingerol }(17.22 \%) \text { and } \\
\text { Shogaol }(0.72 \%) \\
\alpha \text {-glucosidase inhibition } \\
\text { anti-inflammatory }\end{array}$ & $\begin{array}{c}\text { AGI: IC50=1/4 980.21 } \\
\text { mg/ml } \\
\text { Anti-inflammatory: IC50 = } \\
145.04 \mathrm{mg} / \mathrm{ml}\end{array}$ & - & [69] \\
\hline $\begin{array}{l}\text { Methanolic } \\
\text { Extraction }\end{array}$ & Obese mice & \multirow[t]{2}{*}{$\begin{array}{l}\text { Improve insulin sensitivity } \\
\text { Decrease insulin levels }\end{array}$} & $\begin{array}{l}\text { Blood glucose } 20.6 \% \text { lower } \\
\text { than control }\end{array}$ & \multirow[t]{2}{*}{$250 \mathrm{mg} / \mathrm{kg}$ p.o. } & \multirow[t]{2}{*}[66]{} \\
\hline $\begin{array}{l}\text { Ethyl Acetate } \\
\text { Extraction }\end{array}$ & Obese mice & & $\begin{array}{l}\text { Blood glucose } 17.8 \% \text { lower } \\
\text { than control }\end{array}$ & & \\
\hline $\begin{array}{l}\text { Aqueous cold } \\
\text { maceration, } \\
\text { Freeze drying }\end{array}$ & $\begin{array}{c}\text { Streptozotocin } \\
\text { induced diabetic rats }\end{array}$ & $\begin{array}{l}\text { Increase peripheral } \\
\text { utilisation of glucose, } \\
\text { correct impaired liver and } \\
\text { kidney glycolysis, limit } \\
\text { gluconeogenic formation }\end{array}$ & $\begin{array}{c}\text { Blood glucose } 67.85 \% \\
\text { lower by } 30 \text { th day }\end{array}$ & $500 \mathrm{mg} / \mathrm{kg}$ & [70] \\
\hline $\begin{array}{l}\text { Ethanolic } \\
\text { extract }\end{array}$ & $\begin{array}{c}\text { Healthy and } \\
\text { streptozotocin } \\
\text { induced diabetic rats }\end{array}$ & Anti-hyperglycemia & $\begin{array}{l}\text { Maximum blood glucose } \\
\text { reduction of } 50.25 \% \& \& \\
53.14 \% \text { in healthy \& } \\
\text { diabetic rats respectively }\end{array}$ & $800 \mathrm{mg} / \mathrm{kg}$ p.o. & {$[65]$} \\
\hline Ginger juice & $\begin{array}{c}\text { Streptozotocin } \\
\text { induced diabetic rats }\end{array}$ & $\begin{array}{c}\text { Inhibit } \\
\text { 5-HT-induced } \\
\text { hyperglycemia and } \\
\text { hypoinsulinemia } \\
\end{array}$ & $\begin{array}{c}\text { Postprandial blood glucose } \\
23.2 \% \text { lower and insulin } \\
\text { levels } 41.1 \% \text { higher than } \\
\text { diabetic control } \\
\end{array}$ & $4 \mathrm{~mL} \mathrm{~kg}^{-1}$ & {$[64]$} \\
\hline Ginger Powder & $\begin{array}{c}\text { Streptozotocin } \\
\text { induced diabetic rats }\end{array}$ & $\begin{array}{l}\text { Prevent hypoinsulinaemia } \\
\text { and hyperglycemia }\end{array}$ & $\begin{array}{l}\text { Blood glucose } 52 \% \text { lower } \\
\text { and insulin levels } \\
25 \% \text { higher than diabetic } \\
\text { control }\end{array}$ & $\begin{array}{l}200 \mathrm{mg} / \mathrm{kg} \\
\text { body weight }\end{array}$ & [63] \\
\hline $\begin{array}{l}\text { Isolated [6]- } \\
\text { Gingerol from } \\
\text { ethanolic } \\
\text { extract }\end{array}$ & DM type 2 mice & $\begin{array}{c}\text { [6]-Gingerol } \\
\text { Improve glucose tolerance } \\
\text { and inhibit rise of } \\
\text { postprandial glucose levels }\end{array}$ & $\begin{array}{c}\text { Fasting blood glucose } \\
54.8 \% \text { lower than diabetic } \\
\text { control by day } 12 \\
\text { Plasma insulin } \\
\text { concentrations } 46.2 \% \text { lower } \\
\text { than diabetic control by day } \\
12 \\
\text { Blood glucose AUC } 40.5 \% \\
\text { lower than diabetic control } \\
\text { by day } 12\end{array}$ & $\begin{array}{l}100 \mathrm{mg} / \mathrm{kg} \\
\text { body daily }\end{array}$ & [71] \\
\hline $\begin{array}{l}\text { Dried Ginger in } \\
\text { Capsule }\end{array}$ & $\begin{array}{c}32 \text { diabetic Male } \\
\text { Patient (40-60 years } \\
\text { old) }\end{array}$ & Anti-hyperglycemia & $\begin{array}{l}\text { Blood glucose } 17 \% \text { lower } \\
\text { than control }\end{array}$ & $\begin{array}{c}500 \\
\mathrm{mg} / \text { capsule }\end{array}$ & {$[72]$} \\
\hline $\begin{array}{c}\text { Aqueous } \\
\text { Extraction with } \\
\text { heat of: }\end{array}$ & & & & & \multirow[t]{3}{*}[73]{} \\
\hline Fresh Ginger & In vitro & $\alpha$-glucosidase inhibition & IC50(sucrase): $>47 \mathrm{mg} / \mathrm{ml}$ & - & \\
\hline Dried Ginger & In vitro & $\alpha$-glucosidase inhibition & $\begin{array}{l}\text { IC50(sucrase): } 19.61 \mathrm{mg} / \mathrm{ml} \\
\text { IC50(maltase): } 13.38 \mathrm{mg} / \mathrm{ml}\end{array}$ & & \\
\hline $\begin{array}{c}\text { Aquaeous } \\
\text { Maceration of: }\end{array}$ & & & & & \multirow[t]{3}{*}{ [74] } \\
\hline White Ginger & In vitro & $\begin{array}{c}\text { Non-phenolic } \\
\text { phytochemicals } \\
\alpha \text {-amylase inhibition } \\
\alpha \text {-glucosidase inhibition }\end{array}$ & $\begin{array}{l}\text { AAI IC50: } 3.14 \mathrm{mg} / \mathrm{ml} \\
\text { AGI IC50: } 1.68 \mathrm{mg} / \mathrm{ml}\end{array}$ & - & \\
\hline Red Ginger & In vitro & $\alpha$-glucosidase inhibition & $\begin{array}{l}\text { AAI IC50: } 3.51 \mathrm{mg} / \mathrm{ml} \\
\text { AGI IC50: } 2.01 \mathrm{mg} / \mathrm{ml}\end{array}$ & - & \\
\hline $\begin{array}{l}\text { [6] Gingerol } \\
\text { isolated using } \\
\text { ethanol }\end{array}$ & High fat diet mice & Improve insulin sensitivity & $\begin{array}{c}\text { Fasting blood glucose } 52 \% \\
\text { lower and plasma insulin } \\
38 \% \text { lower than untreated } \\
\text { mice (closer to SD) } \\
\text { HOMA-IR index decrease } \\
\text { by } 83.1 \% \text {, }\end{array}$ & $75 \mathrm{mg} / \mathrm{kg}$ & {$[61]$} \\
\hline 6-paradol & $\begin{array}{l}\text { In vitro (Glucose } \\
\text { utilization assay) }\end{array}$ & $\begin{array}{l}\text { Promote glucose utilization } \\
\text { Reduce postprandial } \\
\text { glucose levels }\end{array}$ & $\begin{array}{c}\text { Insulin absent } \\
\text { EC50(3T3-L1 adipocytes): } \\
65.4 \mu \mathrm{M} \\
\text { EC50(C2C12 myotubes): } \\
54.9 \mu \mathrm{M} \\
\text { Insulin present }\end{array}$ & - & {$[75]$} \\
\hline
\end{tabular}




\begin{tabular}{|c|c|c|c|c|c|}
\hline & & & $\begin{array}{c}\text { EC50(3T3-L1 adipocytes): } \\
53.2 \mu \mathrm{M} \\
\text { EC50(C2C12 myotubes): } \\
542 \mu \mathrm{M}\end{array}$ & & \\
\hline 6-shogaol & & & $\begin{array}{c}\text { Insulin absent } \\
\text { EC50(3T3-L1 adipocytes): } \\
63.9 \mu \mathrm{M} \\
\text { EC50(C2C12 myotubes): } \\
26.4 \mu \mathrm{M}\end{array}$ & & \\
\hline & & & $\begin{array}{c}\text { Insulin present } \\
\text { EC50(3T3-L1 adipocytes): } \\
41.5 \mu \mathrm{M} \\
\text { EC50(C2C12 myotubes): } \\
21.5 \mu \mathrm{M}\end{array}$ & & \\
\hline 6-paradol & High fat diet mice & & $\begin{array}{c}\text { Fasting blood glucose } \\
37.6 \% \text { lower than untreated } \\
\text { mice } \\
\text { Postprandial glucose levels } \\
\text { AUC } 28.57 \% \text { lower than } \\
\text { untreated mice }\end{array}$ & $\begin{array}{c}33.75 \\
\mathrm{mg} / \mathrm{kg} / \text { day }\end{array}$ & \\
\hline [6] Gingerol & DM type 2 mice & $\begin{array}{c}\text { Enhance glucose- } \\
\text { stimulated insulin secretion } \\
\text { Increase glucose uptake in } \\
\text { skeletal muscle }\end{array}$ & $\begin{array}{c}\text { Postprandial blood glucose } \\
46.4 \% \text { lower than diabetic } \\
\text { control } \\
\text { Insulin secretion } 40 \% \\
\text { higher than diabetic control }\end{array}$ & $200 \mathrm{mg} / \mathrm{kg}$ & [62] \\
\hline $\begin{array}{l}\text { Isolated [6]- } \\
\text { Gingerol from } \\
\text { ethanolic } \\
\text { extract }\end{array}$ & $\begin{array}{c}\text { High fat high } \\
\text { carbohydrate diet } \\
\text { (HFHC) mice and } \\
\text { standard diet mice } \\
\text { (control) }\end{array}$ & $\begin{array}{c}\text { Increase AMPK } \alpha \\
\text { phosphorylation and total } \\
\text { AMPK } \alpha \text { in skeletal muscle } \\
\text { tissue } \\
\text { Prevent insulin resistance }\end{array}$ & $\begin{array}{l}\text { Postprandial blood glucose } \\
31 \% \text { lower than untreated } \\
\text { HFHC mice and } 24.6 \% \\
\text { lower than control } \\
\text { Circulating insulin levels } \\
58.8 \% \text { lower than untreated } \\
\text { HFHC mice } \\
\text { HOMA-IR } 71.7 \% \text { lower } \\
\text { than untreated HFHC mice }\end{array}$ & $200 \mathrm{mg} / \mathrm{kg}$ & [60] \\
\hline $\begin{array}{l}\text { Dried Ginger in } \\
\text { Capsule }\end{array}$ & DM type 2 patients & $\begin{array}{c}\text { Reduce CRP and } \mathrm{PGE}_{2} \\
\text { levels } \\
\text { Improve insulin sensitivity } \\
\text { Reduce insulin levels }\end{array}$ & $\begin{array}{c}\text { Minor reduction in FPG, } \\
\text { insulin levels } 44.6 \% \text { lower, } \\
\text { HOMA-IR } 54.8 \% \text { lower } \\
\text { than pre-treatment }\end{array}$ & $\begin{array}{l}\text { 800mg capsule } \\
\text { twice a day } \\
\text { before lunch } \\
\text { and dinner } \\
\end{array}$ & [59] \\
\hline
\end{tabular}

Table 4 Ginger toxicology results

\begin{tabular}{|c|c|c|c|}
\hline Toxicology & Toxic Level & Model & Reference \\
\hline LD50: $4525.5 \mathrm{mg} / \mathrm{kg}$ & $\begin{array}{c}\text { LD50: }>50<500 \\
\mathrm{mg} / \mathrm{kg}\end{array}$ & $\begin{array}{l}\text { Streptozotocin induced diabetic } \\
\text { rats }\end{array}$ & {$[70]$} \\
\hline LD50: $1551 \pm 75 \mathrm{mg} / \mathrm{kg}$ & $\begin{array}{c}\text { LD50: }>50<500 \\
\mathrm{mg} / \mathrm{kg}\end{array}$ & Healthy mice & [65] \\
\hline $\begin{array}{l}\text { Gingerol at } 1 \mathrm{ng} / \mathrm{ml} \text { to } 100 \mu \mathrm{g} / \mathrm{ml} \text { did not affect cell viability of } \\
\text { 3T3-L1 cells }\end{array}$ & - & 3T3-L1 pre-adipocytes cells & [71] \\
\hline $\begin{array}{c}\text { Minor gastrointestinal upsets, including eructation, } \\
\text { heartburn, and indigestion }\end{array}$ & - & 27 healthy humans & {$[76]$} \\
\hline LD50: $10.25 \mathrm{~g} / \mathrm{kg} \mathrm{BW}$ (ethanolic maceration) & \multirow{2}{*}{$\begin{array}{c}\text { LD50: }>50<500 \\
\mathrm{mg} / \mathrm{kg}\end{array}$} & Healthy mice & \multirow[t]{2}{*}[77]{} \\
\hline $\begin{array}{l}\text { LD50: } 11.75 \mathrm{~g} / \mathrm{kg} \mathrm{BW} \\
\text { (Aqueous maceration) }\end{array}$ & & & \\
\hline 6-gingerol LD50: $250 \mathrm{mg} / \mathrm{kg} \mathrm{BW}$ & \multirow{2}{*}{$\begin{array}{c}\text { LD50: }>50<500 \\
\mathrm{mg} / \mathrm{kg}\end{array}$} & Healthy mice & \multirow[t]{2}{*}{ [78] } \\
\hline Shogaol LD50: $687 \mathrm{~g} / \mathrm{kg} \mathrm{BW}$ & & & \\
\hline
\end{tabular}

\section{CONCLUSION}

Constantly unmanaged blood sugar levels lead to high circulating insulin levels which may have an impact on skin health and also reduce insulin sensitivity over time leading to poor blood sugar regulation. Hyperglycemia caused by poor blood glucose levels regulation leads to increased risks in CVD, PCOS and type 2 diabetes and there are certain individuals who are at a higher risk of developing 
these diseases. Not only to prevent diseases, improved glycemic control can also benefit skin health, mood, and energy levels. Hence, controlling blood sugar levels is able to benefit healthy individuals as well.

Lemongrass and ginger are proven able to lower blood sugar levels safely alongside having a comparable efficacy with common pharmaceutical medicine. Other than that lemongrass and ginger are also able to normalize insulin levels and improve insulin sensitivity.

\section{ACKNOWLEDGMENTS}

Special thanks to Swiss German University's staff and lecturers who helped in the making of this paper.

\section{REFERENCES}

[1] P. V Röder, B. Wu, Y. Liu, and W. Han, "Pancreatic regulation of glucose homeostasis," Exp. Mol. Med., vol. 48, no. 3, pp. e219-e219, Mar. 2016, doi: 10.1038/emm.2016.6.

[2] A. Felman, "What should my blood glucose level be?," Medical News Today, 2019. https://www.medicalnewstoday.com/articles/ 249413 (accessed Apr. 05, 2021).

[3] American Diabetes Association, "Good to Know: All About Insulin Resistance," Clin. Diabetes, vol. 36, no. 3, pp. 263-264, Jul. 2018, doi: 10.2337/cd18-0038.

[4] Mi. Mouri and M. Badireddy, "Hyperglycemia," StatPearls [Internet], 2020.

[5] Diabetes Care, "Diagnosis and Classification of Diabetes Mellitus," Diabetes Care, vol. 37, no. Supplement 1, p. S81 LP-S90, Jan. 2014, doi: $10.2337 / \mathrm{dc} 14-\mathrm{S} 081$.

[6] CDC, "What is diabetes?," Centers for Disease Control and Prevention, 2020. .

[7] A. Semeco, "15 Easy Ways to Lower Blood Sugar Levels Naturally," Healthline, 2020. .

[8] J. J. Marín-Peñalver, I. Martín-Timón, C. Sevillano-Collantes, and F. J. Del CañizoGómez, "Update on the treatment of type 2 diabetes mellitus," World J. Diabetes, vol. 7, no. 17 , pp. 354-395, Sep. 2016, doi: 10.4239/wjd.v7.i17.354.

[9] D. P. P. R. Group, "Long-term effects of lifestyle intervention or metformin on diabetes development and microvascular complications over 15-year follow-up: the
Diabetes Prevention Program Outcomes Study," lancet Diabetes Endocrinol., vol. 3, no. 11, pp. 866-875, 2015.

[10] D. Delima, L. Widowati, Y. Astuti, H. Siswoyo, R. Gitawati, and A. Purwadianto, "Gambaran praktik penggunaan jamu oleh dokter di enam provinsi di Indonesia,” 2019.

[11] G. Melappa, "Diabetes \& Metabolism A Review on Role of Plant(s) Extracts and its Phytochemicals for the Management of Diabetes," J. Diabetes Metab., vol. 06, Jul. 2015, doi: 10.4172/2155-6156.1000565.

BPOM, "PERATURAN BADAN PENGAWAS OBAT DAN MAKANAN NOMOR 34 TAHUN 2019 TENTANG KATEGORI PANGAN.” BPOM, 2019, Accessed: Jun. 05, 2021. [Online]. Available: https://jdih.pom.go.id/download/product/827/ $34 / 2019$

[13] K. L. Breymeyer, J. W. Lampe, B. A. McGregor, and M. L. Neuhouser, "Subjective mood and energy levels of healthy weight and overweight/obese healthy adults on high-and low-glycemic load experimental diets," Appetite, vol. 107, pp. 253-259, 2016.

[14] E. B. Levitan, Y. Song, E. S. Ford, and S. Liu, "Is nondiabetic hyperglycemia a risk factor for cardiovascular disease?: a meta-analysis of prospective studies," Arch. Intern. Med., vol. 164, no. 19, pp. 2147-2155, 2004.

[15] J. R. L. Batubara, “Acanthosis Nigricans dan Hubungannya dengan Resistensi Insulin pada Anak dan Remaja," Sari Pediatr., vol. 12, no. 2, pp. 67-73, 2016.

[16] R. Kuroki et al., "Acanthosis nigricans with Severe Obesity, Insulin Resistance and Hypothyroidism: Improvement by Diet Control," Dermatology, vol. 198, no. 2, pp. 164-166, 1999, doi: 10.1159/000018096.

[17] A. Romo and S. Benavides, "Treatment Options in Insulin Resistance ObesityRelated Acanthosis Nigricans," Ann. Pharmacother., vol. 42, no. 7-8, pp. 10901094, May 2008, doi: 10.1345/aph.1K446.

[18] R. N. Smith, N. J. Mann, A. Braue, H. Mäkeläinen, and G. A. Varigos, "The effect of a high-protein, low glycemic-load diet versus a conventional, high glycemic-load diet on biochemical parameters associated with acne vulgaris: A randomized, investigator-masked, controlled trial," J. Am. Acad. Dermatol., vol. 57, no. 2, pp. 247-256, 2007. 
[19] D. Deplewski and R. L. Rosenfield, "Growth Hormone and Insulin-Like Growth Factors Have Different Effects on Sebaceous Cell Growth and Differentiation1," Endocrinology, vol. 140, no. 9, pp. 4089-4094, Sep. 1999, doi: 10.1210/endo.140.9.6957.

[20] R. N. Smith, A. Braue, G. A. Varigos, and N. J. Mann, "The effect of a low glycemic load diet on acne vulgaris and the fatty acid composition of skin surface triglycerides," $J$. Dermatol. Sci., vol. 50, no. 1, pp. 41-52, 2008.

[21] F. González, "Inflammation in Polycystic Ovary Syndrome: underpinning of insulin resistance and ovarian dysfunction," Steroids, vol. 77, no. 4, pp. 300-305, Mar. 2012, doi: 10.1016/j.steroids.2011.12.003.

[22] J. K. Lee and A. D. Smith, "Metformin as an adjunct therapy for the treatment of moderate to severe acne vulgaris," Dermatol. Online J., vol. 23, no. 11, 2017.

[23] A. Savic-Radojevic et al., "Effect of hyperglycemia and hyperinsulinemia on glutathione peroxidase activity in non-obese women with polycystic ovary syndrome," Hormones, vol. 14, no. 1, pp. 101-108, 2015, doi: 10.14310/horm.2002.1525.

[24] F. Shishehgar, P. Mirmiran, M. Rahmati, M. Tohidi, and F. Ramezani Tehrani, "Does a restricted energy low glycemic index diet have a different effect on overweight women with or without polycystic ovary syndrome?," $B M C$ Endocr. Disord., vol. 19, no. 1, p. 93, 2019, doi: 10.1186/s12902-019-0420-1.

[25] L. Morin-Papunen et al., "Metformin Improves Pregnancy and Live-Birth Rates in Women with Polycystic Ovary Syndrome (PCOS): A Multicenter, Double-Blind, Placebo-Controlled Randomized Trial," $J$. Clin. Endocrinol. Metab., vol. 97, no. 5, pp. 1492-1500, May 2012, doi: 10.1210/jc.20113061.

[26] Y.-Y. Zhang, L.-Q. Hou, and T.-Y. Zhao, "Effects of acarbose on polycystic ovary syndrome: a meta-analysis," Exp. Clin. Endocrinol. Diabetes, vol. 122, no. 06, pp. 373-378, 2014

[27] J. C. Marshall and A. Dunaif, "Should all women with PCOS be treated for insulin resistance?," Fertil. Steril., vol. 97, no. 1, pp. 18-22, Jan. 2012, doi: 10.1016/j.fertnstert.2011.11.036.

[28] E. Diamanti-Kandarakis and A. Dunaif,
"Insulin Resistance and the Polycystic Ovary Syndrome Revisited: An Update on Mechanisms and Implications," Endocr. Rev., vol. 33, no. 6, pp. 981-1030, Dec. 2012, doi: 10.1210/er.2011-1034.

[29] I. Verkouter et al., "The Association between Adult Weight Gain and Insulin Resistance at Middle Age: Mediation by Visceral Fat and Liver Fat," J. Clin. Med., vol. 8, no. 10, p. 1559, Sep. 2019, doi: 10.3390/jcm8101559.

[30] B. B. Kahn and J. S. Flier, "Obesity and insulin resistance," J. Clin. Invest., vol. 106, no. 4, pp. 473-481, 2000.

[31] D. E. Kelley, B. Goodpaster, R. R. Wing, and J.-A. Simoneau, "Skeletal muscle fatty acid metabolism in association with insulin resistance, obesity, and weight loss," Am. J. Physiol. Metab., vol. 277, no. 6, pp. E1130E1141, 1999.

[32] C. W. Chia, J. M. Egan, and L. Ferrucci, “Agerelated changes in glucose metabolism, hyperglycemia, and cardiovascular risk," Circ. Res., vol. 123, no. 7, pp. 886-904, 2018.

[33] D. Elahi and D. C. Muller, "Carbohydrate metabolism in the elderly," Eur. J. Clin. Nutr., vol. 54, no. 3, pp. S112-S120, 2000, doi: 10.1038/sj.ejcn.1601032.

[34] A. E. Mathews et al., "Older adults' perceived physical activity enablers and barriers: a multicultural perspective," J. Aging Phys. Act., vol. 18, no. 2, pp. 119-140, 2010.

[35] I. Consortium et al., "The link between family history and risk of type 2 diabetes is not explained by anthropometric, lifestyle or genetic risk factors: the EPIC-InterAct study," Diabetologia, vol. 56, no. 1, pp. 60-69, Jan. 2013, doi: 10.1007/s00125-012-2715-x.

[36] S. C. Hardianti, D. I. Widiputri, and M. D. P. T. G. Puteri, "Process Development for The Production of Cymbopogon Citratus and Zingiber Officinale Roscoe Liquid Extracts in Herbal Industry," Swiss German University, 2020 .

[37] H. A. Garba, A. Mohammed, M. A. Ibrahim, and M. N. Shuaibu, "Effect of lemongrass (Cymbopogon citratus Stapf) tea in a type 2 diabetes rat model," Clin. Phytoscience, vol. 6, pp. 1-10, 2020.

[38] C. E. Ekpenyong, K. Davies, and E. E. Antai, "Cymbopogon Citratus stapf (DC) extract ameliorates atherogenic cardiovascular risk in Diabetes-induced Dyslipidemia in rats," $J$. 
Adv. Med. Med. Res., pp. 4695-4709, 2014.

[39] A. J. Ademuyiwa and O. K. Grace, "The effects of Cymbopogon citratus (Lemongrass) on the antioxidant profiles wistar albino rats," Merit Res. J. Environ. Sci. Toxicol. (ISSN 2350-2266), vol. 3, no. 4, pp. 51-58, 2015.

[40] S. K. Bharti, A. Kumar, O. Prakash, S. Krishnan, and A. K. Gupta, "Essential oil of cymbopogon citratus against diabetes: Validation by In vivo experiments and computational studies," J. Bioanal. Biomed., vol. 5, no. 5, pp. 194-203, Dec. 2013, doi: 10.4172/1948-593X.1000098.

[41] M. Bacanl1 et al., "D-limonene ameliorates diabetes and its complications in streptozotocin-induced diabetic rats," Food Chem. Toxicol., vol. 110, pp. 434-442, 2017.

BPOM, "PERATURAN BADAN PENGAWAS OBAT DAN MAKANAN NOMOR 32 TAHUN 2019 TENTANG PERSYARATAN KEAMANAN DAN MUTU OBAT TRADISIONAL." Badan Pengawas Obat dan Makanan (BPOM), pp. 2 3, 2019, [Online]. Available: https://asrot.pom.go.id/asrot/index.php/downl oad/dataannounce2/204/PerBPOM 32 Tahun 2019 Persyaratan dan Keamanan Mutu OT.pdf.

[43] A. A. Adeneye and E. O. Agbaje, "Hypoglycemic and hypolipidemic effects of fresh leaf aqueous extract of Cymbopogon citratus Stapf. in rats," J. Ethnopharmacol., vol. 112, no. 3, pp. 440-444, 2007.

[44] T. Jumepaeng, S. Prachakool, D. Luthria, and S. Chanthai, "Determination of antioxidant capacity and $\alpha$-amylase inhibitory activity of the essential oils from citronella grass and lemongrass," Int. Food Res. J., vol. 20, pp. 1337-1341, Jan. 2013.

[45] M. D. P. T. Gunawan-Puteri, F. Rustandi, and P. Hendra, "Spray Dried Aqueous Extract of Lemongrass (Cymbopogon citratus) exhibits In Vitro and In Vivo Anti Hyperglycemic Activities," J. Farm. Sains dan Komunitas (Journal Pharm. Sci. Community), vol. 15, no. 2, pp. 55-61, 2018.

[46] P. Soewondo, A. Ferrario, and D. L. Tahapary, "Challenges in diabetes management in Indonesia: a literature review," Global. Health, vol. 9, p. 63, Dec. 2013, doi: 10.1186/1744-8603-9-63.
B. M. Josopandojo, M. D. P. T. G. Puteri, and
D. I. Widiputri, "Development Of Food Ingredients With Antidiabetic Activities From Lemongrass (Cymbopogon Citratus)," 2016.

[48] F. M. Tjiptadi, M. D. P. T. Gunawan-Puteri, and Z. Udin, "'In vitro evaluation of active fraction(s) from lemongrass (Cymbopogon citratus) for alpha glucosidse inhibitors', Tangerang Selatan: Postgraduate Swiss German University.," Swiss German University, 2017.

[49] F. Santoso, J. Sunardi, F. Ignatia, and M. D. P. T. Gunawan-Puteri, "THE IMPACTS OF FORMULATION AND STORAGE ON $\alpha$ GLUCOSIDASE INHIBITORY ACTIVITY OF LEMONGRASS, GINGER, AND BLACK TEA FUNCTIONAL BEVERAGES," J. Pharm. Sci. Community, vol. 18, no. 1, pp. 26-38, 2021, doi: 10.24071/JPSC.002637.

[50] A. Susanto, M. D. P. T. G. Puteri, and N. Artanti, "Impact of Lemongrass (Cymbopogon Citratus) Extract Preservation Method to the Alpha-Glucosidase Inhibitory Activity and Chemical Contents," Swiss German University, 2019.

[51] N. Abbas, M. Hamed Al-Sueaadi, A. Rasheed, and E. S. Ahmed, "STUDY OF ANTIDIABETIC EFFECT OF LEMONGRASS (CYMBOPOGON CITRATUS) AQUEOUS ROOTS AND FLOWER EXTRACTS ON ALBINO MICE," Int. J. Pharm. Sci. Res., vol. 9, no. 8, pp. 3552-55, 2018, doi: 10.13040/IJPSR.0975-8232.9(8).3552-55.

[52] N. K. K. Boaduo, D. Katerere, J. N. Eloff, and V. Naidoo, "Evaluation of six plant species used traditionally in the treatment and control of diabetes mellitus in South Africa using in vitro methods," Pharm. Biol., vol. 52, no. 6, pp. 756-761, Jun. 2014, doi: 10.3109/13880209.2013.869828.

[53] C. O. Ewenighi et al., "Estimation of lipid profile and glucose level in alloxan-induced diabetic rats treated with Cymbopogon citratus (lemongrass).," J. Exp. Integr. Med., vol. 3, no. 3, 2013.

[54] T. A. More, B. R. Kulkarni, M. L. Nalawade, and A. U. Arvindekar, "Antidiabetic activity of linalool and limonene in streptozotocininduced diabetic rat: a combinatorial therapy approach," Int J Pharm Pharm Sci, vol. 6, no. 8, pp. 159-163, 2014.

[55] M. Najafian, A. Ebrahim-Habibi, P. 
Yaghmaei, K. Parivar, and B. Larijani, "Citral as a potential antihyperlipidemic medicine in diabetes: a study on streptozotocin-induced diabetic rats," J. Diabetes Metab. Disord., vol. 10, p. 3, 2011.

[56] E. Walum, "Acute oral toxicity.," Environ. Health Perspect., vol. 106, no. suppl 2, pp. 497-503, 1998.

[57] D. L. J. Opdyke, "Monographs on fragrance raw materials," Food Cosmet. Toxicol., vol. 13, no. 4, pp. 449-457, 1975.

[58] M. Tsuji et al., "Studies on d-limonene, as gallstone solubilizer (I): general pharmacological studies," Oyo Yakuri, vol. 8, no. 10, pp. 1439-1459, 1974.

[59] T. Arablou, N. Aryaeian, M. Valizadeh, F. Sharifi, A. Hosseini, and M. Djalali, "The effect of ginger consumption on glycemic status, lipid profile and some inflammatory markers in patients with type 2 diabetes mellitus," Int. J. Food Sci. Nutr., vol. 65, Feb. 2014, doi: 10.3109/09637486.2014.880671.

[60] Y. Li, V. H. Tran, B. P. Kota, S. Nammi, C. C. Duke, and B. D. Roufogalis, "Preventative effect of Zingiber officinale on insulin resistance in a high-fat high-carbohydrate diet-fed rat model and its mechanism of action," Basic Clin. Pharmacol. Toxicol., vol. 115, no. 2, pp. 209-215, 2014.

[61] C. Sampath, M. R. Rashid, S. Sang, and M. Ahmedna, "Specific bioactive compounds in ginger and apple alleviate hyperglycemia in mice with high fat diet-induced obesity via Nrf2 mediated pathway," Food Chem., vol. 226, pp. 79-88, 2017.

[62] M. Bin Samad et al., "[6]-Gingerol, from Zingiber officinale, potentiates GLP-1 mediated glucose-stimulated insulin secretion pathway in pancreatic $\beta$-cells and increases RAB8/RAB10-regulated membrane presentation of GLUT4 transporters in skeletal muscle to improve hyperglycemi," $B M C$ Complement. Altern. Med., vol. 17, no. 1, pp. 1-13, 2017.

[63] H. R. Madkor, S. W. Mansour, and G. Ramadan, "Modulatory effects of garlic, ginger, turmeric and their mixture on hyperglycaemia, dyslipidaemia and oxidative stress in streptozotocin-nicotinamide diabetic rats," Br. J. Nutr., vol. 105, no. 8, pp. 1210$1217,2011$.

[64] S. P. Akhani, S. L. Vishwakarma, and R. K.
Goyal, "Anti-diabetic activity of Zingiber officinale in streptozotocin-induced type I diabetic rats," J. Pharm. Pharmacol., vol. 56, no. 1, pp. 101-105, 2004.

[65] J. A. O. Ojewole, "Analgesic, antiinflammatory and hypoglycaemic effects of ethanol extract of Zingiber officinale (Roscoe) rhizomes (Zingiberaceae) in mice and rats," Phyther. Res. An Int. J. Devoted to Pharmacol. Toxicol. Eval. Nat. Prod. Deriv., vol. 20, no. 9, pp. 764-772, 2006.

[66] R. K. Goyal and S. V Kadnur, "Beneficial effects of Zingiber officinale on goldthioglucose induced obesity," Fitoterapia, vol. 77, no. 3, pp. 160-163, 2006.

[67] S. Saensouk and T. Chumroenphat, 6-gingerol content of ginger (Zingiber officinale Roscoe) by different drying metthods. 2018.

[68] BPOM, “Cek Produk BPOM,” BPOM, 2021. .

[69] M. Rani, K. Padmakumari, B. Sankarikutty, L. Cherian, N. V M, and R. K G, "Inhibitory potential of ginger extracts against enzymes linked to type 2 diabetes, inflammation and induced oxidative stress," Int. J. Food Sci. Nutr., vol. 62, pp. 106-110, Mar. 2011, doi: 10.3109/096374866.2010.515565.

[70] N. Abdulrazaq, M. Cho, N. Win, R. Zaman, and M. Rahman, "Beneficial effects of ginger (Zingiber officinale) on carbohydrate metabolism in streptozotocin-induced diabetic rats," Br. J. Nutr., vol. 108, pp. 1194-1201, Dec. 2011, doi: 10.1017/S0007114511006635.

[71] A. Singh, Akanksha, N. Singh, R. Maurya, and A. Srivastava, "Anti-hyperglycaemic, lipid lowering and anti-oxidant properties of [6]gingerol in db/db mice," Int J Med Med Sci, vol. 1, Jan. 2009.

[72] B. Andallu, B. Radhika, and V. Suryakantham, "Effect of aswagandha, ginger and mulberry on hyperglycemia and hyperlipidemia," Plant Foods Hum. Nutr., vol. 58, no. 3, pp. 1-7, 2003.

[73] J. Sunardi, F. Santoso, and M. D. P. T. G. Puteri, "Development Of A Ready-To-Drink Functional Beverage With A-Glucosidase Inhibitory Activity From Black Tea, Lemongrass (Cymbopogon Citratus) And Ginger (Zingiber Officinale Roscoe)," Swiss German University, 2018.

[74] G. Oboh, A. Adefegha, A. J. Akinyemi, and A. O. Ademiluyi, "Inhibitory effects of aqueous 
extract of two varieties of ginger on some key enzymes linked to type-2 diabetes in vitro," $J$. Food Nutr. Res., vol. 49, no. 1, pp. 14-20, 2010.

[75] C.-K. Wei et al., "6-paradol and 6-shogaol, the pungent compounds of ginger, promote glucose utilization in adipocytes and myotubes, and 6-paradol reduces blood glucose in high-fat diet-fed mice," Int. J. Mol. Sci., vol. 18, no. 1, p. 168, 2017.

[76] S. M. Zick et al., "Pharmacokinetics of 6Gingerol, 8-Gingerol, 10-Gingerol, and 6Shogaol and Conjugate Metabolites in Healthy Human Subjects," Cancer Epidemiol. Biomarkers \&amp;amp; Prev., vol. 17, no. 8, pp. 1930 LP - 1936, Aug. 2008, doi: 10.1158/1055-9965.EPI-07-2934.

[77] M. A. Shalaby and A. R. Hamowieh, "Safety and efficacy of Zingiber officinale roots on fertility of male diabetic rats," Food Chem. Toxicol., vol. 48, no. 10, pp. 2920-2924, 2010.

[78] M. Suekawa, A. ISHIGE, K. YUASA, K. SUDO, M. ABURADA, and E. HOSOYA, "Pharmacological studies on ginger. I. Pharmacological actions of pungent constituents,(6)-gingerol and (6)-shogaol," $J$. Pharmacobiodyn., vol. 7, no. 11, pp. 836-848, 1984. 